\title{
Análise epidemiológica das notificações de violência contra crianças e adolescentes no Brasil de 2009 a 2017
}

\author{
Epidemiological analysis of violence notifications against children and adolescents in \\ Brazil from 2009 to 2017
}

Análisis epidemiológico de notificaciones de violencia contra niños y adolescentes en Brasil de 2009 a 2017

Luciana Medeiros Paungartner $^{1 *}$, Julliane Quevedo de Moura², Morgana Thaís Carollo Fernandes ${ }^{3}$, Tiago Sousa Paiva ${ }^{1}$.

\begin{abstract}
RESUMO
Objetivo: Analisar as notificações de violência contra crianças e adolescentes no Brasil de 2009 a 2017. Métodos: Pesquisa quantitativa epidemiológica, descritiva e documental, por meio de acesso aos registros públicos do SINAN/DATASUS. Foram extraídos dados de notificações de violência contra crianças e adolescentes, utilizando os filtros: perfil sociodemográfico (idade entre 0 a 19 anos de idade, sexo, raça/cor), tipo de violência e vínculo com o agressor. Resultados: Foram notificados 645.393 casos de violência no período avaliado, predominantemente do sexo feminino 393.149 (60,97\%). Na faixa etária de 1 a 4 anos o tipo de violência mais comum foi negligência $54.439(8,44 \%)$ e na faixa etária de 15 a 19 anos prevaleceu a violência física $176.670(27,37 \%)$. Predominou a violência contra a raça/cor branca $242.064(37,51 \%)$ e parda $237.464(36,79 \%)$. No que diz respeito ao vínculo do agressor com a vítima, os pais foram os mais recorrentes $259.055(40,13 \%)$ das notificações. Conclusão: Os achados do presente estudo, poderão subsidiar gestores públicos na formulação de ações de saúde que auxiliem a mitigar situações de violência contra crianças e adolescentes. Considera-se necessário o fomento de estratégias para a articulação dos profissionais com essa ferramenta, assim contribuindo para atenuar as subnotificações.
\end{abstract}

Palavras-chave: Violência, Criança, Adolescente, Saúde pública.

\begin{abstract}
Objective: To analyze reports of violence against children and adolescents in Brazil from 2009 to 2017. Methods: Quantitative epidemiological, descriptive and documentary research, through access to public records of SINAN / DATASUS. Data on notifications of violence against children and adolescents were extracted using the filters: socio-demographic profile (age between 0 to 19 years of age, sex, race / color), type of violence and bond with the aggressor. Results: 645,393 cases of violence were reported in the period evaluated, predominantly female, $393,149(60.97 \%)$. In the age group 1 to 4 years old, the most common type of violence was negligence $54,439(8.44 \%)$ and in the age group 15 to 19 years old, physical violence prevailed $176,670(27.37 \%)$. Violence against the race / white was the color predominated $242,064(37.51 \%)$ and brown $237,464(36.79 \%)$. Regarding the aggressor's bond with the victim, the parents were the most recurrent $259,055(40.13 \%)$ of notifications. Conclusion: The findings of the present study may support public managers in the formulation of health actions that help to mitigate situations of violence against children and adolescents. It is necessary to promote strategies for the articulation of professionals with this tool, thus contributing to mitigate underreporting.
\end{abstract}

Keywords: Violence, Child, Adolescent, Public health.

${ }^{1}$ Centro Universitário Ritter dos Reis (UniRitter), Porto Alegre - RS. *E-mail: lumpaungartner@gmail.com

2 Pontifícia Católica do Rio Grande do Sul (PUC-RS), Porto Alegre - RS.

${ }^{3}$ Universidade Federal do Rio Grande do Sul (UFRGS), Porto Alegre - RS. 


\section{RESUMEN}

Objetivo: Analizar las notificaciones de violencia contra niños y adolescentes en Brasil de 2009 a 2017. Métodos: Investigación cuantitativa epidemiológica, descriptiva y documental, mediante del acceso a los registros públicos de SINAN/DATASUS. Fueron extraídos los datos de las notificaciones de violencia contra niños y adolescentes, mediante los filtros: perfil sociodemográfico (edad entre 0 y 19 años, sexo, raza/color), tipo de violencia y vínculo con el agresor. Resultados: fueron reportados 645.393 casos de violencia en el período evaluado, predominantemente del sexo femenino 393.149 (60,97\%). En el grupo de edad de 1 a 4 años, el tipo de violencia más común fue la negligencia 54.439 (8,44\%) y en el grupo de edad de 15 a 19 años, prevaleció la violencia física 176.670 (27,37\%). Predominó la violencia contra la raza/color blanco $242.064(37,51 \%)$ y pardo $237.464(36,79 \%)$. En cuanto al vínculo del agresor con la víctima, los padres fueron los más recurrentes 259.055 (40,13\%) de las notificaciones. Conclusion: Los resultados del presente estudio pueden apoyar a los administradores públicos en la formulación de acciones de salud que ayuden a mitigar situaciones de violencia contra niños y adolescentes. Se considera necesario promover estrategias para la articulación de profesionales con esta herramienta, contribuyendo así a ablandar el subregistro.

Palabras clave: Violencia, Niño, Adolescente, Salud pública.

\section{INTRODUÇÃO}

A violência é um fenômeno complexo e multifacetado, de configuração peculiar derivada das interações sociais, pessoais, políticas e culturais, além de ser compreendida como um objeto cultural naturalizado (MINAYO, MCS e SOUZA ER, 1999). De acordo com a Organização Mundial de Saúde (OMS) conceitua-se como o uso intencional de força física ou poder real ou como ameaça contra si próprio, outra pessoa, um grupo ou uma comunidade, que resulte ou tem grande probabilidade de resultar em ferimentos, morte, danos psicológicos, desenvolvimento prejudicado ou privação (OMS, 2014). Para Sociedade Brasileira de Pediatria (SBP) e OMS crianças comumente são vítimas dos pais e cuidadores e na adolescência, sofrem com bullying, violência sexual e agressão, normalmente praticadas entre colegas e parceiros íntimos (OMS, 2016; SBP, 2018).

A violência física é caracterizada por ato violento com uso da força física, intencional, podendo ser consumada por tapas, beliscões, arremesso de objetos, entre outros. A violência psicológica é todo ato que coloca em risco a autoestima, identidade ou desenvolvimento da criança ou adolescente, a sexual é considerada como manipulação ou estimulação de conotação sexual, que tencione obter satisfação sexual; podendo compreender as relações homo ou heterossexuais. Tratando-se de situações consideradas casos de negligência, essas se caracterizam pela omissão de cuidados dos pais e responsáveis, inclusive institucionais, ao deixarem de fornecer os cuidados básicos para o desenvolvimento físico, psicológico e social das crianças e adolescentes (BRASIL, 2010; OMS, 2015). No Brasil a Constituição de 1.988 conferiu para a família sociedade e ao Estado o compromisso de proteção integral à criança e ao adolescente, assim como nos anos 90, a criação do Estatuto da Criança e do Adolescente (ECA). No final da década de 90 a violência passou a ser assumida também como responsabilidade no setor de saúde, uma vez que ela afeta a saúde, produz doença, podendo ocasionar a morte ou a sua possibilidade próxima (MINAYO MCS e SOUZA ER, 1998; GONÇALVES HS e FERREIRA AL, 2002; MINAYO MCS, 2006).

A Portaria n¹04/2.011, do Ministério da Saúde (MS) prevê a obrigatoriedade da Notificação Compulsória, a fim de comunicar novos casos de doenças e agravos. A notificação se faz uma ferramenta necessária para compreender o perfil epidemiológico da violência, possibilitando ações de prevenção do problema e nos serviços de saúde deve ser feita sempre que houver suspeita ou confirmação de casos. A falta de informações técnicas e científicas, faz permanecer a subnotificação, bem como a dificuldade de identificar a violência nas comunidades (GARBIN CAS, 2015). Cerca de 60\% dos países não possui dados reais de registro dos casos de violência, o que afeta diretamente o trabalho de intervenção e prevenção. No Brasil, o Sistema de Vigilância de Violências e Acidentes (VIVA), implantado em 2006, gera informações sobre violências e acidentes e possui dois componentes, o VIVA/INQUÉRITO e o VIVA/SINAN instrumentos que notificam a violência doméstica, sexual, e/ou outras violências interpessoais e autoprovocadas (BRASIL, 2013). 
A literatura tem apontado o crescente e expressivo cenário envolvendo a violência dirigida contra crianças e adolescentes no Brasil (RATES SMM, et al., 2015; MARTINS AG e NASCIMENTO ARA, 2017; BRASIL, 2020). Em 2019, o relatório anual do Disque Direitos Humanos registrou 86.837 denúncias de violação dos direitos das crianças e dos adolescentes no Brasil, revelando um aumento de 13,9\% no número de denúncias em relação ao ano anterior, apresentando curva ascendente desde 2016 (BRASIL, 2020).

Crianças e adolescentes que sofreram maus-tratos têm risco elevado de na vida adulta, tornarem-se perpetradoras ou até mesmo suscetíveis a outras violências. Desse modo, entende-se que urge a necessidade de mover esforços para a compreensão do fenômeno, bem como para a capacitação de profissionais da saúde que trabalham em contextos que perpassam o diagnóstico da violência contra crianças e adolescentes (ZANELATTO PF, et al., 2012; MARTINS AF, et al., 2013, OMS, 2014).

O objetivo deste estudo foi analisar as notificações epidemiológicas de violência contra crianças e adolescentes no Brasil de 2009 a 2017.

\section{MÉTODOS}

Trata-se de uma pesquisa quantitativa epidemiológica descritiva e documental, baseada nas notificações de violência contra crianças e adolescentes do Sistema de Informação de Agravos de Notificação (SINAN), disponível nos registros públicos da base de dados do departamento de informática do Sistema Único de Saúde do Brasil (DATASUS). O período de coleta foi de 2009 a 2017, a escolha deste recorte temporal se deu para que se pudesse observar a evolução das notificações antes e após sua obrigatoriedade a partir de 2011.

As variáveis extraídas foram: i) características sociodemográficas das vítimas (faixa etária, sexo, raça/cor, região da unidade federativa de moradia); ii) tipo de violência; iii) local de ocorrência; iv) vínculo com o agressor; v) perfil do agressor (a). A conferência foi realizada por dois tabuladores disponibilizados pelo Ministério da Saúde. Foi utilizado o tabulador TabNET, para o cruzamento de variáveis básicas diretamente na internet, e o TabWIN, para tabulações mais avançadas sobre os arquivos capturados.

Os aspectos éticos foram respeitados, visto que os arquivos do SINAN/SUS são de domínio público, divulgados pelo Ministério da Saúde, cuja configuração preserva a identificação dos sujeitos, garantindo a confidencialidade, assim de acordo com a Resolução 510/2.016, não foi necessário a avaliação pelo Conselho Nacional de Saúde e Comissão Nacional de Ética em Pesquisa (CONEP), para pesquisas que utilizam informações de acesso público (BRASIL, 2016). Após levantamento dos dados, os resultados foram sintetizados em tabelas e discutidos a luz da literatura científica.

\section{RESULTADOS}

Foram notificados 645.393 casos de violência contra crianças e adolescentes na faixa etária de 0 a 19 anos no Brasil entre 2009 e 2017. A região Sudeste teve o maior número de notificações perfazendo 268.159 $(41,55 \%)$, seguida da região Sul $147.216(22,81 \%)$.

Quanto a faixa etária prevaleceu entre 15 a 19 anos e 10 a 14 anos, com $241.878(37,48 \%)$ e 162.302 $(25,15 \%)$, respectivamente. O sexo feminino foi o mais frequente em todas as idades $393.149(60,92 \%)$, especialmente de 10 a 14 anos de idade, 112.988 (17,51\%) e 15 a 19 anos, 150.713 (23,35\%), destaca-se que nessas faixas etárias o sexo feminino representou quase o dobro de notificações.

No que diz respeito a raça/cor, 242.064 (37,51\%) das vítimas eram brancas e 237.464 (36,79\%) pardas. $\mathrm{Na}$ estratificação dessa variável por faixa etária, constatou-se que de 10 a 14 anos a raça/cor parda representou o maior número de notificações 64.609 (39,81\%), em todas as outras faixas etárias, a raça/cor branca foi a mais vitimada, demais dados demográficos estão detalhados na Tabela 1.

Entre os locais de ocorrência das agressões, a maior frequência foi na própria residência das vítimas contabilizando $356.069(55,17 \%)$ registros. Dos tipos de violência informados, a física prevaleceu em 323.252 
$(50,09 \%)$ casos, sendo $176.670(27,37 \%)$ na faixa etária de 15 a 19 anos, seguido da violência psicológica/moral com $133.499(20,68 \%)$ casos.

Quanto a natureza das agressões, as lesões autoprovocadas representaram 67.219 dos casos $(10,42 \%)$, principalmente entre jovens de 15 a 19 anos de idade. Foram notificados $161.556(25,03 \%)$ casos de violência sexual principalmente de 10 a 14 anos de idade perfazendo 64.017 (9,92\%). A negligência e abandono, representou $163.269(25,30 \%)$ dos casos, sendo mais comum de 0 a 4 anos de idade $54.439(8,44 \%)$, conforme Tabela 2.

Quanto ao vínculo com a vítima, a mãe foi reportada como a agressora mais frequente em 150.368 $(23,30 \%)$ dos casos, seguido do pai em 108.687 (16,84\%) notificações. Na faixa etária de 15 a 19 anos a própria pessoa foi registrada na ficha de notificação compulsória como autora das lesões autoprovocadas $42.545(6,59 \%)$, nas demais faixas etárias os pais foram os principais agressores, conforme detalhado na Tabela 3.

Foi mais comum a prática de violência contra crianças na faixa etária de 1 a 4 anos, sendo a mãe a principal agressora 46.541(30,95\%), seguida do pai das vítimas 30.061(27,66\%). Padrasto e madrasta juntos, foram mencionados em aproximadamente $5 \%$ das notificações. Houve suspeita de uso de álcool pelo agressor em $95.182(14,75 \%)$ dos casos.

Ao se analisar a evolução dos registros de notificações de violência na faixa etária entre 0 e 19 anos de idade no período analisado percebe-se que a violência apresentou crescimento ascendente, destacando-se o triênio de 2015 a 2017 principalmente no sexo feminino com 78.483 notificações. Figura 1.

Figura 1 - Evolução temporal das notificações de violência contra crianças e adolescentes no Brasil segundo faixa etária e sexo entre 2009 e 2017.

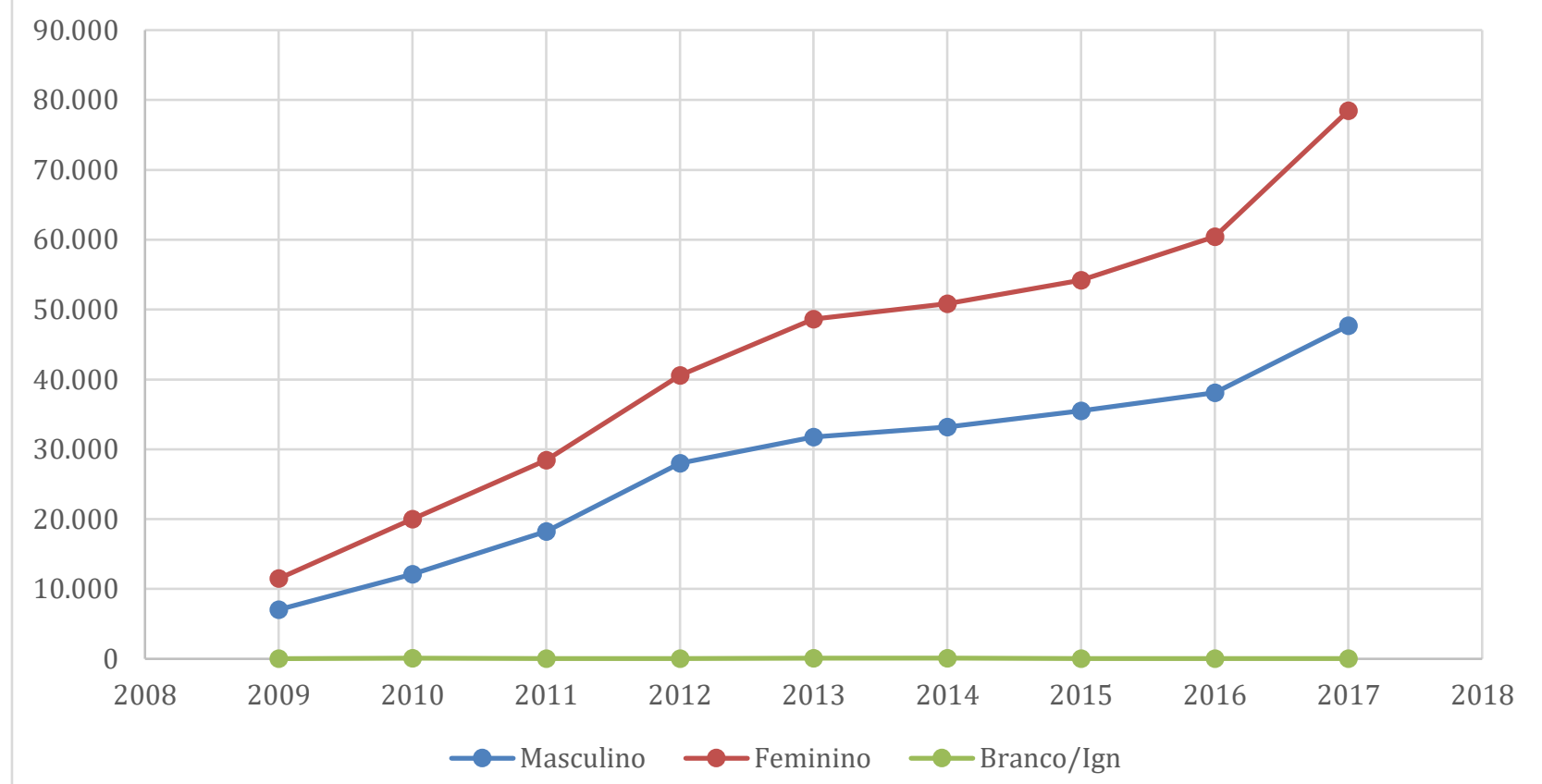

Fonte: Paungartner LM, et al., 2020. Baseado em Ministério da Saúde, Secretaria de Vigilância em Saúde, Sistema de Informação de Agravos de Notificação (SINAN, DATASUS). 


\section{Revista Eletrônica Acervo Saúde / Electronic Journal Collection Health | ISSN 2178-2091}

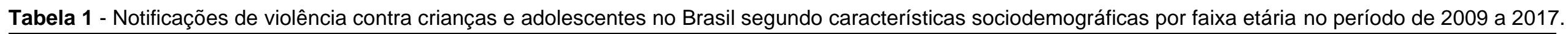

\begin{tabular}{|c|c|c|c|c|c|c|c|c|c|c|c|c|}
\hline \multirow{3}{*}{ Variáveis } & \multicolumn{12}{|c|}{ Faixa etária } \\
\hline & \multicolumn{2}{|c|}{$<1$ ano } & \multicolumn{2}{|c|}{1 a 4 anos } & \multicolumn{2}{|c|}{5 a 9 anos } & \multicolumn{2}{|c|}{10 a 14 anos } & \multicolumn{2}{|c|}{15 a 19 anos } & \multicolumn{2}{|c|}{ Total } \\
\hline & $\mathbf{N}$ & $\%$ & $\mathbf{N}$ & $\%$ & $\mathbf{N}$ & $\%$ & $\mathbf{N}$ & $\%$ & $\mathbf{N}$ & $\%$ & $\mathbf{N}$ & $\%$ \\
\hline \multicolumn{13}{|l|}{ Sexo } \\
\hline Feminino & 25.993 & 50,53 & 53.321 & 54,17 & 50.134 & 54,88 & 112.988 & 69,62 & 150.713 & 62,31 & 393.149 & 60,92 \\
\hline Masculino & 24.973 & 48,55 & 45.080 & 45,80 & 41.205 & 45,11 & 49.302 & 30,38 & 91.146 & 37,68 & 251.706 & 39,00 \\
\hline Ign/branco & 472 & 0,92 & 24 & 0,02 & 11 & 0,01 & 12 & 0,01 & 19 & 0,01 & 538 & 0,08 \\
\hline \multicolumn{13}{|l|}{ Raça/Cor } \\
\hline Branca & 20.647 & 40,14 & 39.704 & 40,34 & 34.886 & 38,19 & 57.804 & 35,62 & 89.023 & 36,80 & 242.064 & 37,51 \\
\hline Parda & 16.238 & 31,57 & 34.493 & 35,04 & 33.525 & 36,70 & 64.609 & 39,81 & 88.599 & 36,63 & 237.464 & 36,79 \\
\hline Preta & 2.481 & 4,82 & 5.107 & 5,19 & 6.313 & 6,91 & 12.682 & 7,81 & 18.386 & 7,60 & 44.969 & 6,97 \\
\hline Indígena & 465 & 0,90 & 605 & 0,61 & 696 & 0,76 & 1.454 & 0,90 & 2.162 & 0,89 & 5.382 & 0,83 \\
\hline Amarela & 209 & 0,41 & 383 & 0,39 & 502 & 0,55 & 1.106 & 0,68 & 1.578 & 0,65 & 3.778 & 0,59 \\
\hline Ign/branco & 11.398 & 22,16 & 18.133 & 18,42 & 15.428 & 16,89 & 24.647 & 15,19 & 42.130 & 17,42 & 111.736 & 17,31 \\
\hline \multicolumn{13}{|l|}{ Região } \\
\hline Nordeste & 10.026 & 19,49 & 17.030 & 17,30 & 12.561 & 13,75 & 26.520 & 16,34 & 49.005 & 20,26 & 115.142 & 17,84 \\
\hline Norte & 2.171 & 4,22 & 7.073 & 7,19 & 9.561 & 10,47 & 20.292 & 12,50 & 16.896 & 6,99 & 55.993 & 8,68 \\
\hline Sudeste & 17.812 & 34,63 & 38.183 & 38,79 & 36.476 & 39,93 & 65.343 & 40,26 & 110.345 & 45,62 & 268.159 & 41,55 \\
\hline Sul & 16.195 & 31,48 & 25.273 & 25,68 & 23.801 & 26,05 & 36.389 & 22,42 & 45.558 & 18,84 & 147.216 & 22,81 \\
\hline Centro-Oeste & 5.234 & 10,18 & 10.866 & 11,04 & 8.951 & 9,80 & 13.758 & 8,48 & 20.074 & 8,30 & 58.883 & 9,12 \\
\hline
\end{tabular}

Legenda: Dados expressos em frequência absoluta e relativa. Ign/branco: registros não informados na ficha de notificação compulsória.

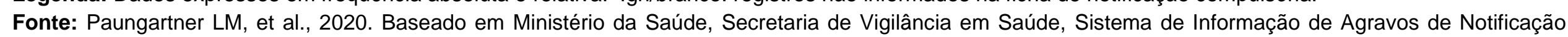
(SINAN, DATASUS). 
Tabela 2 - Notificações de violência contra crianças e adolescentes segundo tipo de violência e local de ocorrência por faixa etária no período de 2009 a 2017.

\begin{tabular}{|c|c|c|c|c|c|c|c|c|c|c|c|c|}
\hline \multirow{4}{*}{ Variáveis } & \multicolumn{12}{|c|}{ Faixa etária } \\
\hline & \multicolumn{2}{|c|}{$<1$ ano } & \multicolumn{2}{|c|}{1 a 4 anos } & \multicolumn{2}{|c|}{5 a 9 anos } & \multirow{2}{*}{\multicolumn{2}{|c|}{$\begin{array}{c}10 \text { a } 14 \text { anos } \\
162.302 ; 25,15\end{array}$}} & \multicolumn{2}{|c|}{15 a 19 anos } & \multicolumn{2}{|c|}{ Total } \\
\hline & 51.438 & 7,97 & 98.425 & & & & & & & & 645.393 & 100 \\
\hline & $\mathbf{N}$ & $\%$ & $\mathbf{N}$ & $\%$ & $\mathbf{N}$ & $\%$ & $\mathbf{N}$ & $\%$ & $\mathbf{N}$ & $\%$ & $\mathbf{N}$ & $\%$ \\
\hline \multicolumn{13}{|l|}{ Lesão autoprovocada } \\
\hline Sim & 946 & 1,84 & 1.536 & 1,56 & 1.029 & 1,13 & 17.833 & 10,99 & 45.875 & 18,97 & 67.219 & 10,42 \\
\hline Não & 46.716 & 90,82 & 90.098 & 91,54 & 85.373 & 93,46 & 122.135 & 75,25 & 166.103 & 68,67 & 510.425 & 79,09 \\
\hline Ian/Em branco & 3.776 & 7.34 & 6.791 & 6.9 & 4.948 & 5,42 & 22.334 & 13,76 & 29.900 & 12,36 & 67.749 & 10.5 \\
\hline \multicolumn{13}{|l|}{ Violência física } \\
\hline Sim & 13.909 & 27,04 & 24.881 & 25,28 & 32.548 & 35,63 & 75.244 & 46,36 & 176.670 & 73,04 & 323.252 & 50,09 \\
\hline Não & 33.073 & 64,3 & 65.814 & 66,87 & 52.798 & 57,8 & 78.529 & 48,38 & 57.807 & 23,9 & 288.021 & 44,63 \\
\hline Ign/Em branco & 4.456 & 8,66 & 7.730 & 7,85 & 6.004 & 6,57 & 8.529 & 5,26 & 7.401 & 3,06 & 34.120 & 5,29 \\
\hline \multicolumn{13}{|l|}{ Viol. psico/Moral } \\
\hline Sim & 4.280 & 8,32 & 13.180 & 13,39 & 24.964 & 27,33 & 41.015 & 25,27 & 50.060 & 20,7 & 133.499 & 20,68 \\
\hline Não & 41.239 & 80,17 & 75.455 & 76,66 & 58.359 & 63,89 & 107.375 & 66,16 & 168.556 & 69,69 & 450.984 & 69,88 \\
\hline Ign/Em branco & 5.919 & 11,51 & 9.790 & 9,95 & 8.027 & 8,79 & 13.912 & 8,57 & 23.262 & 9,62 & 60.910 & 9,44 \\
\hline \multicolumn{13}{|l|}{ Violência sexual } \\
\hline Sim & 2.728 & 5,3 & 26.254 & 26,67 & 38.118 & 41,73 & 64.017 & 39,44 & 30.439 & 12,58 & 161.556 & 25,03 \\
\hline Não & 42.979 & 83,55 & 64.465 & 65,5 & 47.317 & 51,8 & 87.534 & 53,93 & 188.704 & 78,02 & 430.999 & 66,78 \\
\hline Ign/Em branco & 5.731 & 11,14 & 7.706 & 7,83 & 5.915 & 6,48 & 10.751 & 6,62 & 22.735 & 9,4 & 52.838 & 8,19 \\
\hline \multicolumn{13}{|l|}{ Negli/Abandono } \\
\hline Sim & 35.660 & 69,33 & 54.439 & 55,31 & 28.792 & 31,52 & 26.784 & 16,5 & 17.594 & 7,27 & 163.269 & 25,3 \\
\hline Não & 13.336 & 25,93 & 38.422 & 39,04 & 56.097 & 61,41 & 122.326 & 75,37 & 201.047 & 83,12 & 431.228 & 66,82 \\
\hline Ign/Em branco & 2.442 & 4,75 & 5.564 & 5,65 & 6.461 & 7,07 & 13.192 & 8,13 & 23.237 & 9,61 & 50.896 & 7,89 \\
\hline \multicolumn{13}{|l|}{ Local de ocorrência } \\
\hline Residência & 30.409 & 59,12 & 66.206 & 67,27 & 60.910 & 66,68 & 91.751 & 56,53 & 106.793 & 44,15 & 356.069 & 55,17 \\
\hline Habitação coletiva & 195 & 0,38 & 468 & 0,48 & 610 & 0,67 & 1.212 & 0,75 & 1.849 & 0,76 & 4.334 & 0,67 \\
\hline Escola & 371 & 0,72 & 2.698 & 2,74 & 4.656 & 5,10 & 10.921 & 6,73 & 6.321 & 2,61 & 24.967 & 3,87 \\
\hline Prática esportiva & 76 & 0,15 & 134 & 0,14 & 373 & 0,41 & 1.038 & 0,64 & 1.500 & 0,62 & 3.121 & 0,48 \\
\hline Bar ou similar & 315 & 0,61 & 206 & 0,21 & 275 & 0,30 & 1.259 & 0,78 & 6.299 & 2,6 & 8.354 & 1,29 \\
\hline Via pública & 2.767 & 5,38 & 4.189 & 4,26 & 6.682 & 7,31 & 22.868 & 14,09 & 62.979 & 26,04 & 99.485 & 15,41 \\
\hline Com/serviços & 2.275 & 4,42 & 1.525 & 1,55 & 886 & 0,97 & 1.659 & 1,02 & 2.988 & 1,24 & 9.333 & 1,45 \\
\hline Ind/construção & 39 & 0,08 & 52 & 0,05 & 143 & 0,16 & 334 & 0,21 & 524 & 0,22 & 1.092 & 0,17 \\
\hline Outros & 7.727 & 15,02 & 9.866 & 10,02 & 7.286 & 7,98 & 11.581 & 7,14 & 13.348 & 5,52 & 49.808 & 7,72 \\
\hline Ian/Em branco & 7.264 & 14.12 & 13.081 & 13,29 & 9.529 & 10,43 & 19.679 & 12.12 & 39.277 & 16.24 & 88.830 & 13.76 \\
\hline
\end{tabular}

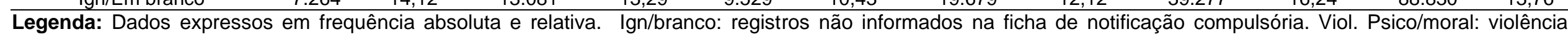

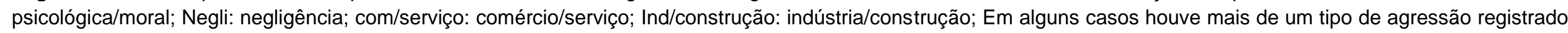

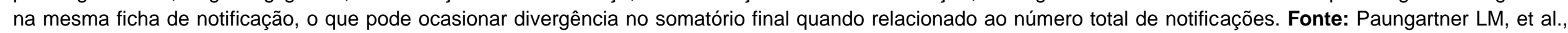
2020. Baseado em Ministério da Saúde, Secretaria de Vigilância em Saúde, Sistema de Informação de Agravos de Notificação (SINAN, DATASUS).

REAS/EJCH | Vol.12(9) | e4241 | DOI: https://doi.org/10.25248/reas.e4241.2020 Página 6 de 11 
Revista Eletrônica Acervo Saúde / Electronic Journal Collection Health | ISSN 2178-2091

Tabela 3 - Notificações de violência contra crianças e adolescentes segundo características do agressor por faixa etária no período de 2009 a 2017.

\begin{tabular}{|c|c|c|c|c|c|c|c|c|c|c|c|c|}
\hline \multicolumn{13}{|c|}{ Faixa etária } \\
\hline \multirow{3}{*}{ Variáveis } & \multicolumn{2}{|c|}{$<1$ ano } & \multirow{2}{*}{\multicolumn{2}{|c|}{$\begin{array}{c}1 \text { a } 4 \text { anos } \\
98.42515,25\end{array}$}} & \multirow{2}{*}{\multicolumn{2}{|c|}{$\begin{array}{c}5 \text { a } 9 \text { anos } \\
91.35014,15\end{array}$}} & \multicolumn{2}{|c|}{10 a 14 anos } & \multicolumn{2}{|c|}{15 a 19 anos } & \multicolumn{2}{|c|}{ Total } \\
\hline & \multicolumn{2}{|c|}{$51.438 \quad 7,97$} & & & & & \multirow{2}{*}{$\begin{array}{c}162.302 \\
N\end{array}$} & \multirow{2}{*}{$\begin{array}{r}25,15 \\
\%\end{array}$} & \multirow{2}{*}{$\frac{241.878}{N}$} & \multirow{2}{*}{$\begin{array}{r}37,48 \\
\%\end{array}$} & \multirow{2}{*}{$\begin{array}{l}645.393 \\
N\end{array}$} & \multirow{2}{*}{$\begin{array}{l}100 \\
\%\end{array}$} \\
\hline & $\mathbf{N}$ & $\%$ & $\mathbf{N}$ & \multirow[t]{2}{*}{$\%$} & \multirow[t]{2}{*}{$\mathbf{N}$} & \multirow[t]{2}{*}{$\%$} & & & & & & \\
\hline \multicolumn{10}{|l|}{ Pai } & & & \\
\hline Sim & 15.567 & 30,26 & 30.061 & 30,54 & 22.261 & 24,37 & 24.159 & 14,89 & 16.639 & 6,88 & 108.687 & 16,84 \\
\hline Não & 27.939 & 54,32 & 52.466 & 53,31 & 57.324 & 62,75 & 116.841 & 71,99 & 178.625 & 73,85 & 433.195 & 67,12 \\
\hline Ign/Em branco & 7.932 & 15,42 & 15.898 & 16,15 & 11.765 & 12,88 & 21.302 & 13,12 & 46.614 & 19,27 & 103.511 & 16,04 \\
\hline \multicolumn{13}{|l|}{ Mãe } \\
\hline Sim & 31.424 & 61,09 & 46.541 & 47,29 & 28.439 & 31,13 & 26.718 & 16,46 & 17.246 & 7,13 & 150.368 & 23,3 \\
\hline Não & 14.138 & 27,49 & 38.295 & 38,91 & 52.135 & 57,07 & 114.837 & 70,76 & 178.383 & 73,75 & 397.788 & 61,64 \\
\hline Ign/Em branco & 5.876 & 11,42 & 13.589 & 13,81 & 10.776 & 11,8 & 20.747 & 12,78 & 46.249 & 19,12 & 97.237 & 15,07 \\
\hline \multicolumn{13}{|l|}{ Padrasto } \\
\hline Sim & 613 & 1,19 & 4.229 & 4,3 & 7.956 & 8,71 & 11.573 & 7,13 & 5.386 & 2,23 & 29.757 & 4,61 \\
\hline Não & 42.196 & 82,03 & 77.662 & 78,9 & 71.081 & 77,8 & 128.742 & 79,32 & 189.334 & 78,28 & 509.015 & 78,87 \\
\hline Ign/Em branco & 8.629 & 16,78 & 16.534 & 16,8 & 12.313 & 13,48 & 21.987 & 13,55 & 47.158 & 19,5 & 106.621 & 16,52 \\
\hline \multicolumn{13}{|l|}{ Madrasta } \\
\hline Sim & 82 & 0,16 & 525 & 0,53 & 788 & 0,86 & 858 & 0,53 & 512 & 0,21 & 2.765 & 0,43 \\
\hline Não & 36.468 & 70,9 & 69.537 & 70,65 & 66.548 & 72,84 & 119.131 & 73,4 & 163.842 & 67,74 & 455.526 & 70,58 \\
\hline Ign/Em branco & 14.888 & 28,94 & 28.363 & 28,82 & 24.014 & 26,29 & 42.313 & 26,07 & 77.524 & 32,05 & 187.102 & 28,99 \\
\hline \multicolumn{13}{|l|}{ Própria pessoa } \\
\hline Sim & 1.497 & 2,91 & 2.628 & 2,67 & 1.719 & 1,88 & 15.705 & 9,68 & 42.545 & 17,59 & 64.094 & 9,93 \\
\hline Não & 42.406 & 82,44 & 81.336 & 82,64 & 78.074 & 85,46 & 126.853 & 78,16 & 161.953 & 66,96 & 490.622 & 76,02 \\
\hline Ign/Em branco & 7.535 & 14,65 & 14.461 & 14,69 & 11.557 & 12,65 & 19.744 & 12,16 & 37.380 & 15,45 & 90.677 & 14,05 \\
\hline
\end{tabular}

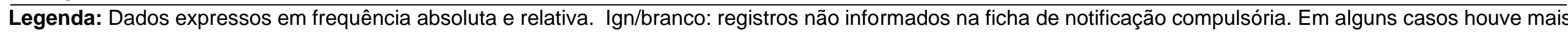

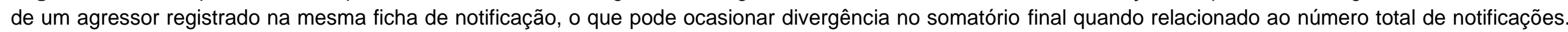

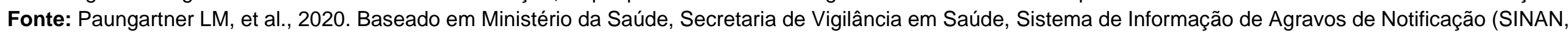
DATASUS). 


\section{DISCUSSÃO}

O presente estudo apresentou um panorama epidemiológico da violência contra crianças e adolescentes no Brasil entre 2009 a 2017 a partir das notificações compulsórias disponíveis no SINAN/DATASUS. Na análise temporal de 2009 a 2017, observou-se uma intensificação do número de notificações de violência contra crianças e adolescentes, a partir de 2011, o que pode sugerir que a obrigatoriedade da notificação a partir deste ano possa ter contribuído para o aumento das notificações mesmo em casos de suspeita de violência.

Quanto ao gênero prevaleceu as notificações de violência perpetrada contra o sexo feminino em todas as faixas etárias analisadas, destacando-se a idade de 10 a 19 anos, em que os casos quando comparados com o sexo masculino duplicaram. Corroborando com o presente estudo, uma pesquisa realizada em um conselho tutelar de Fortaleza - CE, que analisou 840 processos de violência a fim de descrever a natureza das agressões, constatou que as meninas foram vítimas com maior frequência e em $35 \%$ dos casos os pais foram os agressores. Quanto aos tipos, a violência doméstica foi a mais praticada em $62 \%$ dos casos, sendo o próprio domicílio das vítimas o local mais frequente onde ocorreu a violência (MARTINS AF, et al., 2013).

Pesquisa, ainda, analisou a frequência de casos de violência sexual contra crianças e adolescentes no período de 2005 a 2011, notificadas em um centro de referência especializado de assistência social em um município do Nordeste do Brasil e verificou que das 259 notificações, em $89 \%$ dos casos a vítima era do sexo feminino e o principal local de ocorrência foi a própria residência destas, neste estudo os pais e os padrastos eram os principais agressores, além de familiares próximos (OLIVEIRA HC, et al., 2015).

Os dados corroboram os achados desta pesquisa, em que os pais também foram os principais responsáveis pelas agressões, divergindo apenas quanto ao padrasto, que junto com a madrasta representam aproximadamente $5 \%$ dos casos de violência. Ainda, outros estudos também apontaram maior quantitativo de violência praticada contra crianças e adolescentes, sendo mais comum no sexo feminino, ocorridos na residência da vítima (GARBIN CAS, et al., 2011; RATES SMM, et al., 2015; OLIVEIRA HC, et al., 2015; CEZAR PK, ARPINI DM e GOETZ ER, 2017). Ressalta-se que mesmo após o advento da Lei Maria da Penha, lei $n^{\circ} 11.340$ de 2006, criada para coibir e prevenir a violência doméstica e familiar contra mulheres, o índice de violência contra o sexo feminino permanece alto. Ainda que esta lei restrinja à violência doméstica contra a mulher maior e capaz, ela abrange violência familiar da qual podem ser vítimas as crianças e idosos do sexo feminino (BRASIL, 2006).

Quanto ao vínculo com agressor, nesse estudo a mãe foi a principal perpetradora, seguido do pai, principalmente nos casos com faixa etária de 0 a 14 anos. Já entre 14 a 19 anos, prevaleceu a própria pessoa com as lesões autoprovocadas. Um recente estudo em Manaus traçou o perfil da violência contra crianças e adolescentes utilizando dados do SINAN e constatou que os próprios pais das vítimas são responsáveis por $43 \%$ dos casos de violência registrada, sendo também os maiores agressores na faixa de etária de 0 a 9 anos (OLIVEIRA NF, 2020). Com isso, ressalta-se que a violência cometida pelas mães, pais e cuidadores, pode estar associada a práticas corretivas e punitivas (NUNES AJ e SALES, MCV, 2014).

Em relação à mãe ser apontada como a principal agressora, salienta-se que ainda nos moldes patriarcais da sociedade, é a mãe quem geralmente detém o cuidado e responsabilidade dos filhos. Além disso, o ambiente doméstico é favorável à ocorrência da violência, visto que, é nesse espaço que as crianças a maior parte do tempo, e ainda essa população encontra-se em uma etapa do desenvolvimento em que são mais vulneráveis e indefesas (NUNES AJ e SALES MCV, 2014).

A variável raça/cor parda teve o maior registro na faixa etária de 10 a 14 anos de idade e a branca em todas as outras faixas etárias. Corroborando com os achados anteriores, em 2019, o relatório do Disque Direitos Humanos registrou 86.837 denúncias de violação dos direitos das crianças e dos adolescentes, com maior incidência no sexo feminino entre 4 e 11 anos de idade e de raça/cor branca e parda, sendo a negligência a violência mais praticada (BRASIL, 2020).

Estudo baseado na Pesquisa Nacional de Saúde dos Escolares (PENSE) que analisou fatores relacionados a violência doméstica contra adolescentes, participou do inquérito 102.301 alunos, se evidenciou 
que a raça/cor parda teve maior registro de relato de agressões em meninas. A cada sete escolares, um mencionou ter sofrido agressão física por um familiar adulto.

Houve maior chance de relatos de agressão em meninas escolares na faixa etária de treze anos, no grupo que se auto referiu de raça/cor preta, parda e amarela. Aa menor escolaridade da mãe foi associada a maior chance de violência. Esse estudo concluiu que houve um aumento de $36 \%$ na prevalência de agressão contra jovens na faixa etária pesquisada causada por familiares (PENSE, 2015; MALTA DC, et. Al., 2019).

O Ministério da Saúde revela a precocidade de óbitos e violência na população negra. Entre os fatores relacionados estão a falta de acesso à educação, saneamento básico, baixos rendimentos e condições precárias de moradia que são fatores encontrados em maiores índices na população negra e evidencia que o recorte racial está relacionado a desigualdade social no país (BRASIL, 2017). Segundo o Atlas da Violência homens jovens negros constituem o perfil mais frequente de homicídios no Brasil, sendo também os mais expostos a violência de todos os tipos (BRASIL, 2018).

Quanto a natureza das agressões, as lesões autoprovocadas, apresentaram expressivo número na adolescência, entre 15 a 19 anos de idade, podendo sugerir risco para suicídio. Para a OMS o suicídio é considerado uma grande preocupação em saúde pública na sociedade contemporânea. Ainda que no panorama mundial, o Brasil tenha baixos índices de suicídio, tal problemática, é a terceira maior causa de morte na faixa etária entre 15 e 35 anos em ambos os sexos. O suicídio é um problema complexo e plurifacetado, não pode ser relacionado a apenas uma causa ou razão, sendo resultado da interação de fatores (OMS, 2000).

Em relação aos demais tipos de violência, a negligência e abandono, representaram 163.269 (25,30\%) dos casos, sendo mais comum de 0 a 4 anos de idade 54.439 (8,44\%), a física 323.252 mil $(50,09 \%)$, sexual 161.556 mil (25,03\%) e psicológica/moral com 133.449 mil casos $(20,66 \%)$.

Estudo realizado em 2011 com a mesma abordagem metodológica identificou que dos tipos de violência analisados, as predominantes foram a negligência $(n=7.716 ; 47,5 \%)$, seguidas das violências física $(n=$ $5.969,38,5 \%)$, sexual $(n=5.675,37 \%)$ e psicológica/moral $(n=3.772 ; 25,2 \%)$. A negligência foi mais frequente em crianças menores de 0 a 1 ano $(67,8 \% ; p<0,001)$ e a psicológica predominou no grupo de crianças entre 6 a 9 anos $(38,2 \% ; p<0,001)$. (RATES SMM, et al., 2015).

No que diz respeito a suspeita de uso de álcool pelo agressor, a presente pesquisa indicou somente $14,75 \%$ nas notificações indo ao encontro de pesquisa em que os a maioria dos das vítimas alegou não ter suspeitado de que o agressor estava sob o efeito do álcool no momento da agressão (MOREIRA KFA, et al., 2017).

Em 2017 pesquisa bibliográfica realizada entre 2003 e 2013, relacionou a violência doméstica ao uso de álcool e o principal agressor o homem, sendo as principais vítimas as mulheres e as crianças. O homem também é o principal usuário de álcool. $O$ alcoolismo se fez presente como um fator associado à violência (MARTINS AG e NASCIMENTO ARA, 2017).

Estudo que acompanhou pessoas que admitidas em um hospital do Distrito Federal por lesões autoprovocadas e cruzamento dos dados a partir da inclusão no SINAN pela Vigilância Epidemiológica da Secretaria do Estado evidenciou forte subnotificação de dados quanto a tentativa de suicídio e alertou para o não cumprimento por parte dos profissionais da saúde no que se refere a notificação em caso de comportamento suicida, como preconiza o MS (BAÈRE F, 2019).

Por meio dos dados da presente pesquisa, percebe-se o quantitativo demasiado de registros ignorados e em branco apesar da notificação de casos de violência contra crianças e adolescentes ser de suma importância para subsidiar a política de saúde pública no Brasil. Um estudo sobre o conhecimento das enfermeiras hospitalares sobre os aspectos éticos e legais no cuidado de enfermagem às vítimas de violência evidenciou falta de informações entre estes profissionais na realização da notificação compulsória, evidenciando o desconhecimento acerca do tema (ACOSTA DF, et al., 2017). 
A notificação compulsória deve ser compreendida como uma ferramenta primordial para garantia dos direitos e de proteção, subsidiando os profissionais da saúde, educação e assistência social. Notificar traz responsabilidade à todas as esferas da sociedade em prol da proteção das crianças e adolescentes. Entre os obstáculos para o ato de notificar, destacam-se a falta de conhecimento do ECA pelos profissionais, a falta de qualificação para o reconhecimento das situações de risco ou sinais de alerta, além do medo de represálias por parte do agressor (RATES SMM, et al., 2015; CEZAR PK, ARPINI DM e GOETZ ER, 2017; OLIVEIRA BG, et al., 2018).

A violência impacta diretamente a saúde, assim é fundamental que tal problemática possa ser trabalhada por todos os profissionais no campo da saúde, a falta de profissionais preparados a lidar com o tema, tornase um relevante obstáculo a ser vencido (MINAYO MCS, 2016). Os resultados deste estudo estão de acordo com os da literatura, que aponta a violência na infância, especialmente a sexual somente como fenômeno predominantemente intrafamiliar. Nesse sentido, para identificar se a criança está sendo vítima de violência, deve se atentar para sinais como medo, distúrbios psicológicos, agressão, pesadelos, problemas escolares, hiperatividade e comportamento regressivo. Na adolescência, os indícios mais comuns são: depressão, isolamento, comportamento suicida, autoagressão, queixas somáticas, atos ilegais, fugas, abuso de substâncias e comportamento sexual inadequado (CERQUEIRA D, et. al., 2017; PEREZ-FUENTES G, et al., 2013).

Este estudo não se propôs a comparar o número de notificações entre regiões do Brasil, visto a extensão do território de cada região e a relatividade do aspecto populacional, além disso, em cada região a implantação do sistema das vigilâncias de violências encontra-se em um patamar, e é natural que esse processo ocorra singularmente em cada local.

Não obstante, o registro de notificações compulsórias depende do engajamento dos profissionais responsáveis com a ação. Sabe-se que os números apresentados neste e em vários outros estudos, representam uma fração dos casos e estão longe de apresentar os números reais, uma vez que, a subnotificação é um grande desafio a ser enfrentado (BRASIL, 2013).

Há algumas limitações neste trabalho decorrentes da natureza administrativa do banco de dados do SINAN/DATASUS. São possíveis erros de codificação e de preenchimento. Os potenciais vieses decorrentes dessas limitações são reconhecíveis ainda que seja possível obter um panorama das notificações de violência contra crianças e adolescentes ocorridos no Brasil no período de 2009 a 2017. Assim, a relevância de estudos semelhantes a este, devem ser consideradas e estimuladas.

\section{CONCLUSÃO}

Diante dos achados, o objetivo da presente pesquisa foi alcançado, uma vez que, foi possível caracterizar o perfil epidemiológico da violência no Brasil contra crianças e adolescentes entre 2009 e 2017 a partir dos dados viabilizados pelo SINAN/DATASUS. Os achados do presente estudo descritivo podem subsidiar gestores no planejamento de ações preventivas, de programas e políticas de saúde que auxiliam a mitigar situações de violência contra essa população. A escola é um fundamental e imprescindível fator de proteção e deve ser aliada nesse contexto com o Programa Saúde na Escola trabalhando conjuntamente com os profissionais da saúde na construção de diagnósticos e soluções conjuntas no enfrentamento das violências e disseminação da cultura de paz. Não obstante, deve ser lançada mão de estratégias de educação permanente para a articulação dos profissionais com a ferramenta de notificação compulsória contribuindo para atenuar o número de subnotificações, clarificando à gestão pública o real número de casos.

\section{REFERÊNCIAS}

1. ACOSTA DF, et al. Aspectos éticos e legais no cuidado de enfermagem às vítimas de violência doméstica - SC - Brasil. Texto \& Contexto Enfermagem [periódico na Internet]. 2017 ago.

2. BAÈRE F. Registros de tentativas de suicídio no Distrito Federal: Uma realidade subnotificada - DF - Brasil. INTERAÇÃO EM PSICOLOGIA [periódico na Internet]. 2017 Fev-Dez; vol 23 | n 01 | 2019. 
3. BRASIL. Disque 100 [acessado 2020 Jun 6]. Disponível em: https://www.gov.br/mdh/pt-br/acesso-ainformacao/ouvidoria/Relatorio_Disque_100_2019_pdf.

4. BRASIL. Ministério da saúde. Linha de Cuidado para a Atenção Integral à Saúde de Crianças, Adolescentes e suas Famílias em Situação de Violência. Brasília: $1^{\circ}$ edição - Ministério da saúde [periódico na Internet]; 2010.

5. BRASIL. Ministério da saúde. Política Nacional de saúde integral da população negra. Uma política do SUS. Brasília: Ministério da Saúde [periódico na Internet]; 2017; $3^{\circ}$ edição - Ministério da saúde.

6. BRASIL. Ministério da Saúde. Secretaria de Vigilância em Saúde. Departamento de Vigilância de Doenças e Agravos não Transmissíveis e Promoção da Saúde. Brasília: Ministério da Saúde [periódico na Internet]; 2013; ISBN 978-85-334-20229.

7. BRASIL. Decreto-lei n¹1.340 de 7 de agosto de 2006. (Constituição, 2006)

8. BRASIL. Ministério da Saúde. Portaria № 104, de 25 de janeiro de 2011. Função da Lei. Diário Oficial da União, Brasília, DF. Portaria no 104, de 25 de janeiro de 2011.

9. BRASIL. Resolução № 510 de 07 de Abril de 2016.

10. CERQUEIRA D, et al. ATLAS da Violência 2018 - RJ - Brasil. IPEA - INSTITUTO DE PESQUISA ECONÔMICA APLICADA [periódico na Internet]. 2018 Jun.

11. CERQUEIRA D, et al. Estupro no Brasil: Vítimas, autores, fatores situacionais e evolução das notificações no sistema de saúde entre 2011 e 2014, Texto para Discussão, No. 2313, Instituto de Pesquisa Econômica Aplicada (IPEA), Rio de Janeiro, junho de 2017.

12. CEZAR PK, et al. Registros de Notificação Compulsória de Violência Envolvendo Crianças e Adolescentes - DF Brasil. Psicologia: Ciência e Profissão [periódico na Internet]. 2017 Abr-Jun; 37(2), [432-445].

13. GARBIN CAS, et al. Desafios do profissional de saúde na notificação da violência: Obrigatoriedade, efetivação e encaminhamento - RJ - Brasil. Ciência e Saúde Coletiva [periódico na Internet], 2015 Jun; 20(6), [1879-1890].

14. GARBIN CAS, et al. Violência denunciada: ocorrências de maus tratos contra crianças e adolescentes registradas em uma unidade policial - DF - Brasil. Revista Brasileira de Enfermagem [periódico na Internet], 2011 Jul-Ago; 64(4), [665-670].

15. GONÇALVES HS, FERREIRA AL. A notificação da violência intrafamiliar contra crianças e adolescentes por profissionais de saúde - RJ - Brasil. Cadernos de Saúde Pública [periódico na Internet], 2002 Jan-Fev; 18(1), [315-319].

16. MALTA DC, et al. Fatores associados aos episódios de agressão familiar entre adolescentes, resultados da Pesquisa Nacional de Saúde do Escolar (PeNSE) - RJ - Brasil. Ciência \& Saúde Coletiva [periódico na Internet]; 2019 Abr-Mai; 24(4), [1287-1298].

17. MARTINS AF, et al. Violence with children and adolescents: profiles of victims, of aggression and of perpetrators / Violência envolvendo crianças e adolescentes: perfil das vítimas, da agressão e dos agressores - PI - Brasil. Revista de Enfermagem Da UFPI [periódico na Internet], 2013 Out-Dez; 2(4), [50].

18. MARTINS AG, NASCIMENTO ARA. Violência doméstica, álcool e outros fatores associados: uma análise bibliométrica - RJ - Brasil. Arquivos Brasileiros de Psicologia. 2017; 69 (1): [107-121].

19. MINAYO MCS, 2016. Institucionalização do avanços e desafios. Ciência \& Saúde Coletiva, 23(6):2007-2016, 2018.

20. MINAYO MCS, Souza ER de. É possível prevenir a violência? Reflexões a partir do campo da saúde pública - RJ Brasil. Ciência \& Saúde Coletiva [periódico na Internet], 1999; 4(1), [7-23].

21. MINAYO MCS, SOUZA ER. Violência e saúde como um campo interdisciplinar e de ação coletiva - RJ - Brasil. História, Ciências, Saúde - Manguinhos [periódico na Internet], 1997 Nov 1998 Fev; IV(3): [513-531].

22. MINAYO MCS. Violência e saúde [online]. Rio de Janeiro: Editora FIOCRUZ; 2006.

23. MOREIRA KFA, OLIVEIRA DM DE, OLIVEIRA CAB de et al. Perfil das crianças e adolescentes vítimas de violência. Revenferm UFPE online. 2017. DOI: 10.5205/reuol.23542-49901-1-ED.1111201718.

24. NUNES AJ, SALES, MCV, 2014. Violência contra crianças no cenário brasileiro. Ciência \& Saúde Coletiva, 21(3):871-880, 2016

25. OLIVEIRA BG, et al. Responsabilidade dos profissionais de saúde na notificação dos casos de violência - DF - Brasil. Revista Bioética [periódico na Internet]. 2018 Out-Dez; vol. 26 | n³.

26. OLIVEIRA HC, et al. Notificação compulsória de violência sexual contra crianças e adolescentes - BA - Brasil. Arq. Ciênc. Saúde [periódico na Internet]; 2015 Out-Dez; 22(4), [26-30].

27. OLIVEIRA NF, et al., 2020. Violência contra crianças e adolescentes em Manaus, Amazonas: estudo descritivo dos casos e análise completude das fichas de notificação, 2009-2016. Epidemiol. Serv. Saude, Brasília, 29(1):e2018438, 2020

28. ORGANIZAÇÃO MUNDIAL DE SAÚDE. INSPIRE Seven Strategies for Ending Violence Against Children. Organização Mundial de Saúde; 2016; ISBN 9789241565356.

29. ORGANIZAÇÃO MUNDIAL DE SAÚDE. Prevenção do suicídio: um manual para profissionais da saúde em atenção primária - Genebra: Organização Mundial de Saúde [periódico na Internet]. 2000

30. ORGANIZAÇẪO MUNDIAL DE SAÚDE. Prevenindo a violência juvenil: um panorama das evidências. Organização Mundial de Saúde; 2015

31. ORGANIZAÇÃO MUNDIAL DE SAÚDE. Relatório mundial sobre a prevenção da violência. Organização Mundial de Saúde; 2014.

32. PÉREZ-FUENTES G, et. al. Prevalence and correlates of child sexual abuse: a national study. ComprPsychiatry 2013; 54(1):16-27.

33. RATES SMM, et al. Violência infantil: uma análise das notificações compulsórias, Brasil 2011 - RJ - Brasil. Ciência \& Saúde Coletiva [periódico na Internet], 2015 Mar; 20 (3), [655-665].

34. SOCIEDADE BRASILEIRA DE PEDIATRIA - SBP. Sociedade De Pediatria De São Paulo - SPSP ATENDIMENTO ÀS CRIANÇAS E ADOLESCENTES VÍTIMAS DE VIOLÊNCIA. $2^{\underline{a}}$ edição. Brasília: 2018.

35. ZANELATTO PF, et al. Violência Contra Crianças e Adolescentes: Significados e Atitudes por Equipes da Estratégia Saúde da Família - GO - Brasil. Ciencia y Enfermeria [periódico na Internet]; 2012; 18(2), [41-49]. 